\title{
Resonant absorption of electromagnetic fields by surface plasmons buried in a multilayered plasmonic nanostructure
}

\author{
A. P. Hibbins, W. A. Murray, J. Tyler, S. Wedge, W. L. Barnes, and J. R. Sambles \\ Electromagnetic Materials Group, School of Physics, University of Exeter, Exeter EX4 4QL, United Kingdom
}

(Received 30 June 2006; published 22 August 2006)

\begin{abstract}
The optical reflectivity of a metal-dielectric-metal microcavity in which the upper layer is periodically perforated by narrow slits is explored. Complete characterization of the observed modes in terms of their resonant electromagnetic fields is achieved by comparison of the experimental data to the predictions of a finite-element model. In particular, we demonstrate that the slits provide efficient diffractive coupling to a surface plasmon mode buried within the microcavity whose propagation is strongly confined to the dielectric layer.
\end{abstract}

DOI: 10.1103/PhysRevB.74.073408

The texturing of metal surfaces on a subwavelength scale is known to strongly modify their electromagnetic (EM) response. Excitation of surface plasmons (SPs) at these interfaces plays a crucial role in light-matter interactions (see Refs. 1 and 2, and references therein). One of the reasons why the field of plasmonics has received so much attention in recent years is the localization of EM fields associated with these modes, together with their ability to guide energy along interfaces. In addition, metallic microcavity structures have also been explored to provide enhanced performance in a range of optoelectronic devices (e.g., light-emitting $\operatorname{diodes}^{3}$ ). Furthermore, light-guiding structures that allow subwavelength confinement of the optical mode are critical for the achievement of compact photonic components. ${ }^{4,5}$ The design and fabrication of metallic nanostructures combining surface wave properties with microcavity resonant behavior opens up substantial new device potential and has already seen application as ultrathin electromagnetic attenuators in the microwave regime. ${ }^{6}$

In this paper, we study the visible EM response of a metal-dielectric-metal microcavity where the illuminated metal film is perforated with periodically spaced identical slits [Fig. 1(a)]. Both the width of these slits and the thickness of the dielectric core are $\sim \lambda / 10$ (where $\lambda$ is the incident wavelength), the latter being small enough to permit coupling between SPs of the two metal surfaces. The excitation of these coupled-SP modes ${ }^{7}$ using radiation polarized orthogonal to the slits results in resonant absorption of the incident power via Joule heating of the metal. In addition, it is found that single-surface SPs are excited at the illuminated face of the slit array. By recording the positions of the resonances in the reflectivity spectra as a function of the angle of incidence, the band structure of the modes supported is elaborated. The predictions of a finite-element method (FEM) model $^{8}$ are then fitted to the experimental data with good agreement being obtained. Each of the modes supported by the structure is identified by studying the numerically predicted EM field distributions.

To fabricate the sample, a $\mathrm{Ag} / \mathrm{SiO}_{x} / \mathrm{Ag}$ trilayer structure was deposited by vacuum evaporation onto a clean glass slide. The first silver layer is optically thick $(>100 \mathrm{~nm})$, with the thickness of the $\mathrm{SiO}_{x}$ dielectric core $\left(t_{c}\right)$ and upper (illuminated) silver layer $\left(t_{m}\right)$ both somewhat smaller $(\sim 50 \mathrm{~nm})$.
Next the top silver layer was etched by a focused ion beam to mill an array of identical slits covering an area of $\sim 0.75 \mathrm{~mm}^{2}$. This was undertaken in a manner that gave well-formed slits with minimal bridging, while at the same time minimizing the damage to the dielectric core. The width of each slit $(w \sim 50 \mathrm{~nm})$ is significantly less than the visible radiation wavelength, and the periodicity of the array is $\lambda_{g}$ $=340 \mathrm{~nm}$. The choice of these parameters was based upon initial modeling studies that took into account the resolution limitations of the fabrication process together with the requirement to observe well-defined resonances in the visible regime. The geometrical parameters of the structure are determined from scanning electron microscope (SEM) imaging (Table I); an image of a typical region of the sample surface is presented in Fig. 1(b).

The dispersion of the modes is determined by recording the spectra of the specularly reflected beam as a function of the angle of incidence $(\theta)$. A collimated beam from a tungsten halogen lamp was spectrally filtered by a monochromator. The intensity of the incident light was modulated using a mechanical chopper to allow lock-in detection, and a beam splitter redirected a small fraction of the incident light onto a second detector, allowing for source intensity fluctua-
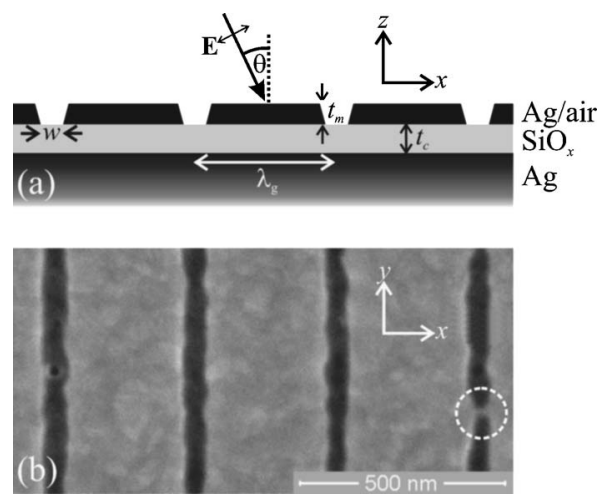

FIG. 1. (a) Schematic of sample structure and coordinate system, where the geometrical parameters are given in Table I. (b) SEM image of a typical region of the sample surface. An example of bridging can be seen in the bottom half of the right-hand slit (circled); on average, such bridging occurs once in every micrometer of slit length. 
TABLE I. Estimates of parameters describing the sample determined from the SEM images. The righthand column gives the parameters used in the numerical model to obtain the "best-fit" comparison with the experimental data.

\begin{tabular}{cccc}
\hline \hline & & SEM imaging & "Best-fit" FEM model \\
\hline Pitch, $\lambda_{g}(\mathrm{~nm})$ & & $340 \pm 3$ & 340 \\
Slit width, $w_{s}(\mathrm{~nm})$ & lower & $55 \pm 5$ & 55 \\
& upper & $\sim 75$ & 75 \\
$\mathrm{SiO}_{x}$ core thickness, $t_{c}(\mathrm{~nm})$ & & $65 \pm 5$ & 70 \\
Upper silver layer thickness, $t_{m}(\mathrm{~nm})$ & & $55 \pm 5$ & 50 \\
SiO permittivity $_{\text {Silver permittivity }}$ & & & $2.2+0 i$ \\
Ref. 9 & at $500 \mathrm{~nm}$ & & $-8.5+0.9 i$ \\
& at $800 \mathrm{~nm}$ & & $-28.0+1.4 i$ \\
\hline \hline
\end{tabular}

tions to be taken into account. Automated variation of $\theta$ was obtained by placing the sample on a motor-driven, computercontrolled rotating stage. Both the sample and polarizer placed in the incident beam were carefully orientated so that transverse magnetic (TM) polarized radiation is incident in the $x z$ plane (Fig. 1). The experimental data (Fig. 2) are recorded as a function of frequency and in-plane wave vector, $k_{\|}$[where $k_{\|}=(2 \pi / \lambda) \sin \theta$ is conserved across the interface], and normalized to a straight-through transmission spectrum in the absence of a sample. The dark bands represent very low reflectivity (resonant absorption) and provide a clear illustration of the band structure of the modes supported by the sample.

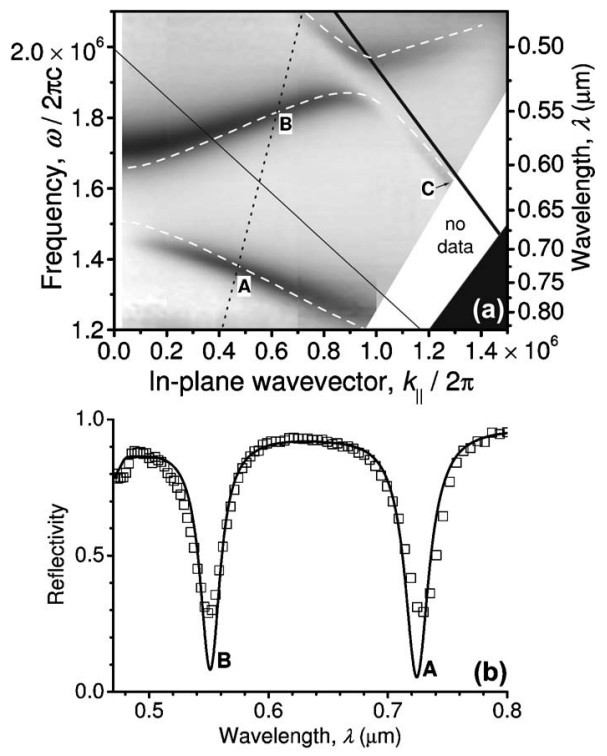

FIG. 2. (a) Dispersion of the modes supported by the structure derived from experimental reflectivity spectra recorded at different angles of incidence and plotted as a function of the in-plane wave vector $\left(k_{\|}=\frac{\omega}{c} \sin \theta\right)$, where dark shading represents low reflectivity. The white dashed lines correspond to the numerically predicted positions of the reflectivity features using the "best-fit" parameter set. The solid black lines represent the diffracted $(+1)$ core (thin) and air (thick) light lines, respectively. (b) Experimental reflectivity spectrum (squares) recorded at $\theta=20^{\circ}$ [section corresponding to the dotted black line in (a)] together with the "best-fit" predictions from the numerical model (solid line).
In order to identify the EM character of each of the modes, a numerical model $^{8}$ was used to predict the reflectivity from the sample. The geometric structure for the model resembles that shown in Fig. 1(a). SEM images of cleaved sections of the structure show that the precise form of slit profile varied somewhat across the sample. However, numerical modeling demonstrates that neither the mode dispersion nor the reflectivity response is affected significantly by the shape variation. Therefore, an average truncated " $\mathrm{V}$ " shape was used to represent all the slits. In fitting the predictions of the model to the recorded data, the grating pitch, the minimum slit width and thicknesses of the upper silver layer, and the dielectric core $\left(\mathrm{SiO}_{x}\right)$ were allowed to vary within their measured bounds. In addition, the real part of the optical permittivity of the $\mathrm{SiO}_{x}$ was used as a fitting parameter, the imaginary part being assumed to be zero. The permittivity of silver was set to recognized frequency-dependent values. ${ }^{9}$ A comparison of the numerically modeled "best-fit" band structure (using the parameters listed in Table I) is compared to the experimental reflectivity data in Fig. 2. We will subsequently show that the bands seen here correspond to the second $(N=2)$ and third $(N=3)$ harmonics of a coupled SP resonance buried within the dielectric core, as well as a single-surface SP on the upper face of the sample. (The fundamental resonance occurs outside of the studied frequency range.) The $\theta=20^{\circ}$ line spectrum taken from this experimental dataset is shown in Fig. 2(b) (squares), and a comparison is made to the numerical predictions (line). Here the intensity of the experimental signal has been corrected and scaled to match the predictions of the off-resonance reflectivity and resonance width, to take into account the incident beam striking some unprocessed regions of the surface and the error in normalization for $\theta>0$.

It is useful at this point to refer the reader to a recent study by the current authors ${ }^{6}$ concerning the microwave reflectivity from a similarly structured but scaled-up sample. The observed reflectivity minima were due to the excitation of resonances of transverse electromagnetic (TEM) modes ${ }^{10}$ within the metal-bound regions of the core. A FEM numerical model was used to calculate the standing-wave field solutions with each mode labeled by an integer $N=n+1$, where $n$ is the number of nodes in the magnetic field within the metal bound regions per repeat period $\left(\lambda_{g}\right)$. For example, the standing-wave state illustrated in Fig. 2(a) of Ref. 6 is the 

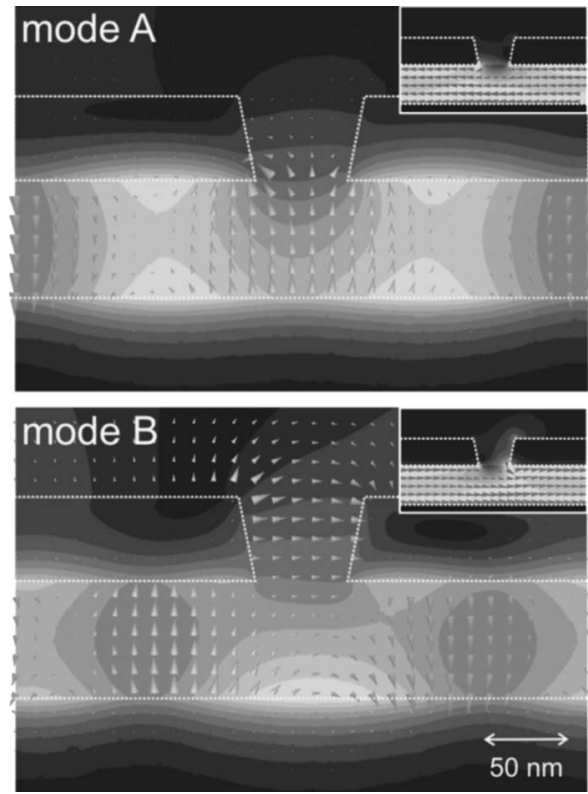

FIG. 3. Predictions from the numerical model of the field distribution of the two modes observed in the $\theta=20^{\circ}$ spectrum (labeled A and $\mathrm{B}$ in Fig. 2). The fields are plotted in the $x z$ plane and one repeat period of the structure is shown $\left(\lambda_{g}\right)$. The gray scale represents the complex magnitude of the magnetic field $|H|(x, y, z)$ where the lightest shading corresponds to field enhancements of at least nine and seven times with respect to the incident field for resonances $\mathrm{A}$ and $\mathrm{B}$, respectively. The arrows illustrate the electric-field vector $[E(x, y, z, t)]$ distribution at an approximate phase corresponding to maximal field intensification. The insets illustrate the magnitude (gray scale) and direction (arrow heads) of the Poynting vector distribution, where the lightest shading corresponds to enhancements of approximately 20 and 40 times compared with the incident field.

fundamental resonance $(N=1)$ since it has no nodes in the magnetic field (antinodes in the electric field) other than directly below the slit. Similarly, the fields shown in Fig. 2(d) correspond to the second harmonic $(N=2)$ with a single magnetic-field node (electric-field antinode) within the metal bound region of the core. Note that while the description of these modes as TEM waves is perfectly valid in the limit of infinite metal conductivity, they can be equivalently and more generally described (i.e., in the visible regime) as two SPs that interact across the dielectric core between the metal layers to form a coupled mode.

Returning to the present work, the EM field distribution for the resonances labeled A, B, and C in Fig. 2 has been calculated. The two main grayscale images of Fig. 3 represent the complex magnitude of the magnetic field $[|H|(x, y, z)]$ associated with resonances labeled $\mathrm{A}$ and $\mathrm{B}$. The data are overlaid with the electric-field vector distribution $[E(x, y, z, t)]$ calculated at a temporal phase corresponding to its maximum enhancement. The insets show the respective Poynting vector distributions. The two bands, which include resonances $\mathrm{A}$ and $\mathrm{B}$, correspond to coupled SPs interacting across the dielectric core with energy propagation strongly confined to this layer. Notice how, owing to the opposite sense of curvature (group velocity) of the bands, the direc-

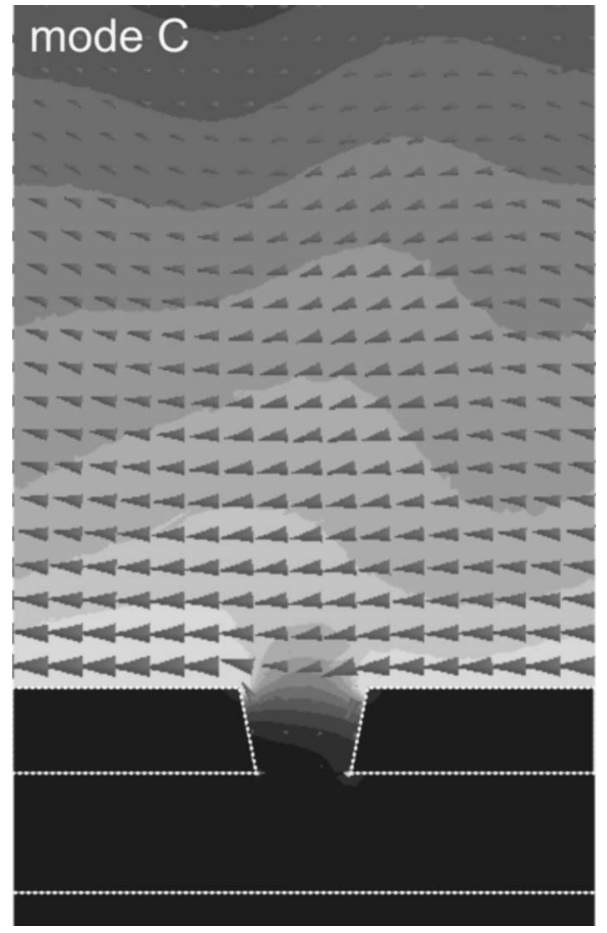

FIG. 4. Numerical predictions of the Poynting vector distribution for the resonance labeled $C$ in Fig. $2(\sin \theta=0.8, \lambda=615 \mathrm{~nm})$. The gray scale and arrowheads correspond to the magnitude and direction, respectively, where the lightest shading represents enhancements of at least 15 times that of the incident field.

tion of power flow for the two modes is in opposite directions. Clearly at $k_{\|}=0(\theta=0)$ the two modes must be standing waves, with the experimental dispersion plot indicating that coupling is then only possible in the upper band. The resonant fields of the upper band edge $(\theta=0)$ closely resemble those illustrated for the same mode at $\theta=20^{\circ}$ (resonance B). This upper band is labeled the third harmonic $(N=3)$ since there are two nodes in the $H$ field within the metal bound regions. Its symmetry dictates that strong coupling will occur at normal incidence since the phase requirement of the incident field at neighboring slits is identical. Even at $\theta=20^{\circ}$, the standing-wave character of the mode is still evident in its resonant field solution but the nature of the mode is mixed. In order to match to the variation in phase of the incident EM fields at the surface, the mode now shows traveling-wave character. On further increment of $\theta$, this upper band interacts and forms an anticrossing with another resonance, whose fields, while not entirely excluded from the core, have strong power flow above and parallel to the upper face of the structure (Fig. 4, resonance C). This mode is a diffractively coupled single-surface SP that without interaction would closely follow the diffracted light line and asymptotically approach the surface plasma frequency in the ultraviolet.

The lower-energy mode, associated with the band gap around $k_{\|}=0$ to which coupling at normal incidence appears impossible, clearly has different character from the upper band-edge mode. The field plots of this band at $\theta=20^{\circ}$ (resonance A, Fig. 3) suggest that within the metal bound region, the standing-wave field exhibits a single node in the $H$ field 
per repeat period, positioned midway between adjacent slits. This is accompanied by an antinode in the $E$ field directly beneath the opening. We therefore label this band as the second harmonic $(N=2)$. TM-polarized radiation incident on the sample induces $E_{x}$ in the slits, and is therefore only able to excite modes that exhibit reversal in the direction of the electric-field vector on either side of the opening at some point in their temporal cycle. Coupling to the lower-energy band is therefore deemed impossible at normal incidence. However as the angle is increased, the phase of the incident field varies across the surface and matching becomes possible in a traveling-wave solution. The frequency of the mode decreases and approaches the diffracted light line of the dielectric core and it is expected to form a band gap with the fundamental $(N=1)$ mode close to a wavelength of $1 \mu \mathrm{m}$ at the Brillouin zone boundary $\left(k_{\|} / 2 \pi \approx 1.5 \times 10^{6} \mathrm{~m}^{-1}\right)$. As a final remark it is insightful to note that, unlike in Ref. 6, a series of standing-wave solutions given by the frequencies $v_{N}=N c /\left(2 \lambda_{g} \sqrt{\varepsilon_{\text {core }}}\right)$ are not expected. This is because at optical frequencies the response function of the silver is far from being that of a perfect conductor, and in addition ideal limits of infinitesimal slit width and core thickness are certainly not realized. ${ }^{11,12}$

In conclusion, it is shown that a metal-clad dielectric film can give very strong resonance absorption in the visible when the upper metallic layer is periodically patterned with narrow slits. By experimentally recording the reflectivity of the structure and comparing the band structure to the predictions of a numerical model, each of the modes supported has been characterized in terms of their EM fields. The second and third harmonics of a coupled SP resonance buried within the dielectric core have been identified, as well as a singlesurface SP on the upper face of the sample. If desired, a more flatbanded response may be engineered by suitable choice of the geometrical parameters of the structure. One could also perforate both metal films with slits to form a narrowband filter, or substitute the dielectric core with a fluorescent layer to yield a novel light source.

The authors acknowledge the financial support of the EPSRC, the EC (under project FP6 NMP4-CT-2003505699), and the Royal Society (under a Wolfson Laboratory refurbishment grant).
${ }^{1}$ W. L. Barnes, A. Dereux, and T. W. Ebbesen, Nature (London) 424, 824 (2003).

${ }^{2}$ J. B. Pendry, L. Martín-Moreno, and F. J. Garcia-Vidal, Science 305, 847 (2004).

${ }^{3}$ E. F. Schubert, N. E. J. Hunt, M. Micovic, R. J. Malik, D. L. Sivco, A. Y. Cho, and G. J. Zydzik, Science 265, 943 (1994).

${ }^{4}$ J. R. Krenn, B. Lamprecht, H. Ditlbacher, G. Schider, M. Salerno, A. Leitner, and F. R. Aussenegg, Europhys. Lett. 60, 663 (2002).

${ }^{5}$ S. A. Maier, P. G. Kik, H. A. Atwater, S. Meltzer, E. Harel, B. E. Koel, and A. A. G. Requicha, Nat. Mater. 2, 229 (2003).

${ }^{6}$ A. P. Hibbins, J. R. Sambles, C. R. Lawrence, and J. R. Brown,
Phys. Rev. Lett. 92, 143904 (2004).

${ }^{7}$ E. N. Economou, Phys. Rev. 182, 539 (1969).

${ }^{8}$ HFSS (Ansoft Corporation, Pittsburgh, PA).

${ }^{9}$ E. D. Palik, Handbook of Optical Constants and Solids (Academic Press, Inc., London, 1985).

${ }^{10}$ R. Collin, Field Theory of Guided Waves, 2nd ed. (Wiley, New York, 1990), Chap. 7.

${ }^{11}$ Y. Takakura, Phys. Rev. Lett. 86, 5601 (2001).

${ }^{12}$ J. R. Suckling, A. P. Hibbins, M. J. Lockyear, T. W. Preist, J. R. Sambles, and C. R. Lawrence, Phys. Rev. Lett. 92, 147401 (2004). 